Cita bibliográfica: Landaluce Calvo, M.I. (2017). Identificación de los perfiles dinámicos provinciales de la demanda turística en España. Un enfoque exploratorio espacio-temporal. Investigaciones Turísticas (14), pp. 6386. http://dx.doi.org/10.14461/INTURI2017.14.04

\title{
Identificación de los perfiles dinámicos provinciales de la demanda turística en España. Un enfoque exploratorio espacio-temporal
}

\author{
Identification of provincial dynamic profiles of tourist demand in Spain. A time-space \\ exploratory approach
}

María Isabel Landaluce Calvo. Universidad de Burgos. España. iland@ubu.es

\section{RESUMEN}

En este trabajo se afronta un estudio exhaustivo del perfil dinámico de la demanda turística provincial en España durante los últimos 18 años. Su originalidad se basa en el doble enfoque "espacio-temporal". Por un lado, se analiza la concentración temporal del flujo turístico y, por otro lado, se define una agrupación en clases de las provincias españolas atendiendo a su trayectoria, encontrando principalmente dinámicas tendenciales y diferentes dinámicas estacionales. El tándem integrado por el Análisis Factorial Múltiple y los Métodos de Clasificación constituye la propuesta metodológica.

Palabras clave: demanda turística provincial; series temporales; análisis factorial; clasificación

\section{ABSTRACT}

In this work we face a comprehensive study of the dynamic profile of the provincial tourist demand in Spain during the last 18 years. The originality of this work is based on the double approach "space-time". On the one hand, we analyze the concentration of the tourist flow and, on the other hand, we define a grouping in some classes of the spanish provinces attending to their trayectories, finding mainly trend dynamics and different seasonal dynamics. The tandem integrated by the Multiple Factor Analysis and the Classification Methods constitutes the methodological proposal.

Keywords: provincial tourist demand, time series, factorial analysis, classification

\section{INTRODUCCIÓN}

En su último informe, el World Travel \& Tourism Council valora en 2,2 billones de dólares el impacto económico (directo) que generó la industria del turismo a nivel mundial en el año 2015 (lo que representa el 3,1\% del PIB); estimando un crecimiento del 3,3\% para el año 2016. Ahora bien, sumando los efectos directos, indirectos e inducidos, la valoración del impacto alcanza los 7,2 billones de dólares, esto es, el 9,8\% del PIB mundial; superando a otros sectores como la industria química $(8,6 \%)$, la agricultura $(8,5 \%)$, la educación $(8,4 \%)$, la automoción (7\%) o la banca (5,9\%). Asimismo, en este informe queda reflejado que 1 de cada 11 puestos de trabajo están, directa o indirectamente, relacionados con la industria turística 
(WTTC, 2016). A nivel nacional, y como puede deducirse del siguiente párrafo, la realidad del sector no es muy distinta.

"El turismo cierra un ejercicio excepcional e imprevisto, con un crecimiento del PIB turístico que revisamos de nuevo por tercera vez hasta el 4,9\%, con la creación de 80668 nuevos empleos al cierre de 2016. Ello supone un $15 \%$ de todos los nuevos empleos creados en España, un aumento de 6414 millones de $€$ de actividad directa e indirecta generada por el Turismo (estimación a partir de la Cuenta Satélite) hasta lograr 125 mil millones de $€$, lo que elevará la participación del turismo al $11,2 \%$ del PIB (...) La actividad turística seguirá creciendo en 2017 pero a menor ritmo (...) Concretamente, estimamos que el PIB turístico crezca un 3,2\% en 2017, de forma que el turismo seguirá siendo uno de los principales motores del crecimiento de la economía española y de la creación de empleo (EXCELTUR, 2016, pp. 4-20."

Estos datos hablan por sí solos de la importancia del sector para el desarrollo económico, en general. Centrándonos en nuestro entorno, se puede afirmar que la industria turística se caracteriza por su solidez y su consolidada posición de liderazgo a nivel internacional, contando además con gran potencial de crecimiento futuro. No obstante, desarrolla su actividad en un entorno económico globalizado, de gran incertidumbre y en el que continuamente surgen nuevas tendencias y retos. Por ello, el Consejo Español de Turismo, con base en esta realidad competitiva y cambiante, acordó llevar a cabo un amplio proceso de revisión estratégica del sector, que se ha materializado en el Plan del Turismo Español Horizonte 2020 (Secretaría General de Turismo, 2007). En esta revisión se incide en el análisis de los flujos turísticos, destacando como seña de identidad su concentración en los meses de verano. No en vano, una de las líneas de acción previstas en el Plan se centra en incrementar la competitividad del sector turístico, fortaleciendo el valor de las experiencias ofrecidas a los turistas y aprovechando las tendencias de la demanda que reduzcan su estacionalidad. Este perfil estacional del turismo nacional conlleva una serie de efectos negativos, tanto económicos como sociales, de los que EXCELTUR, entre otros, se ha hecho eco en reiterados informes. Por ello, debe de ser objeto de importantes esfuerzos por parte de todos los agentes implicados y estar presente, de manera prioritaria, en la planificación y puesta en marcha de sus estrategias de crecimiento futuro.

El mundo académico no se ha mantenido ajeno a esta problemática y ha prestado considerable atención al estudio del turismo, en general, y al perfil estacional de la demanda turística, en particular. En este último contexto, en el ámbito nacional y centrado en la identificación de diferentes perfiles a nivel provincial, se sitúa este trabajo de investigación. El artículo se estructura como sigue. Después de esta breve introducción, se presenta una revisión de la literatura relacionada con el tema tratado, poniendo de manifiesto la originalidad del enfoque metodológico utilizado, al no existir ninguna referencia al respecto. En la sección tercera, se describen brevemente los datos utilizados, su estructuración y unas pinceladas sobre la estrategia de análisis seleccionada como idónea para los objetivos perseguidos. A continuación, en el cuarto apartado, se presentan e interpretan los resultados más destacados, tanto gráficos como numéricos, ilustrando, a su vez, la adecuación de la metodología. En la sección quinta se discuten algunos resultados y en la sexta se exponen las principales conclusiones; reflejando, asimismo, las limitaciones que este trabajo presenta y 
que, por tanto, dejan abiertas otras líneas de investigación futuras. Por último, se aportan las referencias bibliográficas utilizadas.

\section{ESTACIONALIDAD DEL TURISMO. ESTADO DEL ARTE}

La comunidad científica ofrece numerosos y rigurosos estudios relacionados con la Economía del Turismo, en términos globales, y con el perfil estacional de la demanda turística, en términos más concretos. En el artículo de Wanhill (2011) se puede encontrar una selección de trabajos realizados en este campo, distinguidos como "best paper" en la revista Tourism Economics. Pero este es sólo un ejemplo, ya que son varias las revistas, incluidas en el Social Science Citation Index, que han surgido en torno al conocimiento de este sector, con importantes colaboraciones de autores españoles, como reflejan Albacete y Fuentes (2009), entre las que podemos destacar, junto a la ya mencionada, Tourism Management, Annals of Tourism Research, International Journal of Comtemporary Hospitality Management o Journal of Travel Research. En el ámbito nacional, mencionar el libro conmemorativo del 20 aniversario de la Asociación Española de Expertos Científicos, AECIT (Aguiló y Antón, 2015). A lo largo de sus 20 capítulos y con la participación de 27 colaboradores, queda reflejada la versatilidad de la investigación en el sector turístico, así como las inquietudes multidisciplinares que la realidad turística despierta entre investigadores de diferentes universidades y centros repartidos por toda la geografía española. La amplia y actualizada bibliografía que aparece al final de cada uno de los capítulos así lo pone de manifiesto. Uno de sus capítulos, el número 10, está íntegramente dedicado a la estacionalidad y concentración territorial.

En las referencias consultadas parece haber consenso en la identificación del trabajo de Baron (1975) como uno de los primeros estudios científicos del fenómeno de la estacionalidad en el turismo, siguiendo vigente en nuestros días su distinción entre estacionalidad natural (basada en aspectos climatológicos) y estacionalidad institucional (basada en aspectos culturales). Si bien, esta clasificación ha sido ampliada y matizada en trabajos posteriores, surgiendo la conveniencia (o necesidad) de investigar diversos aspectos estrechamente relacionados con este fenómeno, también denominado concentración temporal de la actividad turística. En este sentido, Koenig-Lewis y Bischoff (2005) presentan un exhaustivo estado del arte de la investigación realizada sobre la estacionalidad, y clasifican en seis las líneas de interés tratadas en la literatura: definiciones de la estacionalidad, las causas, los impactos, las implicaciones políticas, el comportamiento de los turistas y las técnicas de medición de la estacionalidad.

En la literatura especializada de autores de nuestro entorno nacional destaca el trabajo de López y López (2007) en el que presentan una revisión de artículos delimitando el ámbito de trabajo a cuatro cuestiones, atendiendo a su mayor actualidad y a su mayor proyección de futuro: factores determinantes de la estacionalidad, los efectos provocados, las estrategias e implicaciones políticas y su medición. Además, en el seno de cada una de estas cuestiones, estos autores identifican diferentes fenómenos y asocian los trabajos referenciados a cada uno de ellos. Así, consideran que son cinco los principales factores determinantes de la estacionalidad: la climatología, las decisiones personales en el ámbito de lo social, religioso o cultural, la presión social o la moda, las temporadas deportivas y la inercia o la tradición. En lo que respecta a los efectos que las variaciones estacionales puedan causar citan cuatro: económicos, laborales, ecológicos y socioculturales. Señalan que las estrategias más utilizadas 
son tres: diversificación del producto, la segmentación del mercado y la diferenciación de precio. El cuarto y último aspecto se refiere a la medición de la estacionalidad, línea de investigación en la que se sitúa este trabajo, por lo que la dedicamos una atención especial.

En Cisneros Martínez y Fernández Morales (2015) se puede encontrar una excelente revisión de la literatura científica, a nivel mundial, referente a los diferentes enfoques utilizados para medir la estacionalidad. Sin embargo, en esta revisión se quiere dejar impronta de los trabajos realizados, a lo largo de las últimas tres décadas, por autores nacionales. Los pioneros, Aguiló y Sastre (1984) propusieron la medición de la estacionalidad, en el caso de Baleares, a través de los coeficientes de variación y de Gini, entendida ésta como desigualdad distribucional de la demanda turística a lo largo del año, incidiendo en la relación de este fenómeno con el desempleo en la región. Ramón y Abellán (1995), con la misma metodología, amplían el análisis de la estacionalidad al territorio nacional. Muñiz (2001), por su parte, utiliza el método X-11 ARIMA para evidenciar las graves consecuencias de la estacionalidad, especialmente en algunas CCAA, $y$, a su vez, presenta el turismo social como una tipología desestacionalizadora. López y López $(2006,2007)$, como ya se ha comentado, recogen una amplia revisión de la literatura sobre este tema, en la que detallan las principales líneas de investigación; en ambos trabajos vuelven a recuperar la metodología de Gini para medir la concentración del mercado turístico, si bien, hay que señalar que el primero de ellos tiene la originalidad de estar enfocado desde la oferta turística. Bujosa y Roselló (2011) ofrecen una óptica original, analizando, a través de un modelo de elección discreta, el efecto estacional del cambio climático sobre la probabilidad de visitar un determinado destino turístico. Riera y otros (2011) se atreven a medir la eficiencia de una muestra de establecimientos de alojamiento turístico en Baleares, a partir de la aplicación del análisis envolvente de datos, explicando parte de las diferencias encontradas en base al patrón estacional de la actividad turística. El trabajo de Duro y Farré (2015), además de recoger referencias actualizadas de la literatura científica sobre el tema, es otro exponente más del uso de las medidas de desigualdad para el análisis descriptivo de la concentración temporal en la demanda turística española; si bien, complementa el uso de los tradicionales coeficientes de variación y de Gini con los índices de la familia de Theil, comprobando que la aplicación de cada uno de ellos puede generar resultados distintos.

El presente trabajo continúa la estela del último artículo referenciado en el párrafo anterior. También se lleva a cabo un análisis de la concentración temporal de la demanda turística en las provincias españolas, a partir de los datos de pernoctaciones hoteleras, ampliando el periodo hasta el año 2016. Sin embargo, se propone un enfoque metodológico distinto, alternativo y complementario al utilizado por estos autores, como va a quedar patente a lo largo de las siguientes secciones.

\section{DATOS, OBJETIVOS y METODOLOGÍA}

La información analizada para estudiar el perfil estacional de la demanda turística en España, siguiendo la filosofía y justificación de Duró y Farré (2015), corresponde al indicador que mide las "pernoctaciones" durante los últimos 18 años (enero 1999 - septiembre 2016). El Instituto Nacional de Estadística (INE), a través de la Encuesta de Ocupación Hotelera, proporciona esta variable desagregada territorialmente para todas las provincias, siendo el mes la unidad temporal de referencia. Por tanto, los datos de partida están constituidos por 52 series con 213 observaciones temporales. 
El objetivo aquí perseguido es doble. Por un lado, visualizar y medir, de forma exhaustiva, los principales patrones presentes en la dinámica turística española, cuantificando las posibles diferencias $y / 0$ las posibles similitudes existentes entre las trayectorias comparadas, correspondientes a cada una de las provincias. Este objetivo no es novedoso, ya que, como se ha señalado en la sección anterior, existen diversos trabajos con esta misma temática. No obstante, y por otro lado, se propone para alcanzar ese objetivo una estrategia de análisis diferente, complementaria, a las utilizadas en los artículos citados en la sección 2. La metodología utilizada se apoya en un enfoque exploratorio de datos de doble naturaleza "espacio-temporal", constituyendo una aportación original de esta investigación.

La estrategia de análisis se basa en el análisis simultáneo de un conjunto de series temporales yuxtapuestas, estructuradas como una tabla de tres dimensiones (también denominada tabla múltiple, ver figura 1). Está integrada por la combinación de una técnica de análisis factorial exploratorio adaptada al tratamiento de datos susceptibles de ser estructurados en varios grupos de variables, Análisis Factorial Múltiple (AFM), y por una técnica de Análisis de Clasificación (CLA) (Lebart et al., 2006). Aun siendo técnicas conocidas en el campo del análisis de datos, con aplicaciones en ámbitos de investigación muy diversos, ninguna de ellas había sido utilizada para el estudio de series temporales, en general, ni para el análisis de la estacionalidad, en particular. En los trabajos de Landaluce (2016 y 2017) se puede encontrar una incipiente línea de investigación en este sentido.

Las tres dimensiones de los datos a las que se hace referencia en el párrafo anterior, son el fenómeno a estudiar, las observaciones temporales y los contextos en los que se mide el fenómeno. En este estudio, se concretan en la demanda turística (pernoctaciones), los meses analizados (desde enero de 1999 hasta septiembre de 2016) y el conjunto de provincias españolas, respectivamente. El análisis exploratorio desde la óptica de tabla múltiple y a través de la conjunción del AFM y de CLA va a permitir contestar, entre otras, a las siguientes preguntas: ¿La dinámica turística, desde el punto de vista de la demanda, presenta un perfil estacional? ¿Esta dinámica ha sido similar en los 52 territorios considerados? ¿Cuáles mantienen las mayores similitudes? ¿Y las mayores diferencias? ¿En qué unidades temporales se han producido estas similitudes y/o diferencias? Esto es, van a quedar identificadas las posibles regularidades, los patrones y/o concentraciones temporales subyacentes en las dinámicas turísticas provinciales, llegando a clasificar las provincias en diferentes tipologías atendiendo a sus trayectorias. Todas las conclusiones alcanzadas estarán avaladas por una amplia batería de indicadores numéricos y de ilustrativos gráficos.

A continuación, se expone esquemáticamente la metodología propuesta. La presentación de las dos técnicas involucradas en este enfoque se realiza a través de unas breves y generales pinceladas, introduciendo la terminología propia de las mismas. Los instrumentos gráficos y los indicadores numéricos asociados serán presentados con mayor detalle en la sección 4, para un mejor seguimiento por los lectores no familiarizados con las técnicas factoriales y/o con el análisis de tablas múltiples. El desarrollo detallado de ambas metodologías, para aquellos que deseen profundizar en las mismas, pueden encontrarse, entre otros trabajos, en Abascal y Grande (2005), Escofier y Pagès $(1986,1992,1994)$ y Lebart et al. (2006). 


\subsection{Análisis Factorial Múltiple (AFM)}

En términos generales, la metodología AFM se puede resumir en los siguientes puntos:

1. Es una técnica que permite el tratamiento simultáneo, como activas, de varias tablas de datos referidas al mismo conjunto de observaciones, pudiendo tener diferente número de variables y de distinta naturaleza (cualitativa o cuantitativa), aunque en el seno de cada una de ellas las variables deben ser de la misma naturaleza. Para las variables continuas, como en esta investigación, el AFM se comporta como un Análisis de Componentes Principales, ACP, (ponderando las variables).

2. Una de sus etapas consiste en abstraer los principales factores de variabilidad de las observaciones, estando estas últimas descritas, de manera equilibrada, por los diversos grupos de variables. Desde este punto de vista, el AFM proporciona los resultados clásicos de los análisis factoriales. Esto es, eje por eje, se obtienen las coordenadas, contribuciones y cosenos cuadrado de las observaciones, así como los coeficientes de correlación entre las variables continuas. Respecto a los métodos factoriales clásicos, el AFM presenta una gran ventaja al ofrecer resultados típicos de la estructura en grupos del conjunto de variables, que van a tener un destacado protagonismo en el estudio comparativo de series temporales.

3. A cada grupo de variables se asocia una nube de observaciones, que recibe el nombre de nube parcial, y que será analizada por separado, a través de un ACP, obteniendo los denominados factores parciales. Esta fase carece de sentido en el caso aquí tratado, ya que se han construido grupos de una sola variable. Así mismo, se define una nube global que resulta de la unión de todas las nubes parciales (previamente ponderadas), de cuyo análisis se obtendrán unos factores globales. Esta nube global tiene asociada una inercia que se denomina inercia total. En este método se descompone esta inercia total, de forma aditiva, en inercia intra e inercia inter.

4. Uno de los principales objetivos del AFM es examinar la existencia de estructuras comunes a todas, o aparte, de las nubes parciales, lo que se reflejaría en una inercia intra mínima o pequeña. $\mathrm{O}$, lo que es lo mismo, dada la descomposición aditiva antes mencionada, en una inercia inter máxima o elevada. Para ello, proporciona una representación superpuesta de estas nubes parciales proyectándolas sobre los ejes del análisis global de todos los grupos, como elementos ilustrativos. Aquéllas observaciones cuyos puntos parciales (puntos que representan a cada observación desde los diferentes grupos) se sitúen próximos (inercia intra débil) ilustran la estructura común de las distintas tablas analizadas. Por el contrario, aquéllas observaciones con puntos parciales asociados alejados unos de otros (inercia intra alta), constituyen las excepciones a la estructura común.

5. El AFM proporciona medidas globales de relación entre los grupos, basadas en el coeficiente de correlación vectorial entre matrices RV de Y. Escoufier (Robert y Escoufier, 1976). Este coeficiente se obtiene a partir de los coeficientes de correlación lineal entre dos variables cualesquiera. Su valor está comprendido entre 0 (no existe relación entre las variables de los dos grupos considerados) y 1 (las nubes que representan a los grupos son homotéticas).

Todos estos aspectos, a nivel práctico y de facilidad de interpretación, se concretan en tres etapas: 
- Etapa de inter-estructura: análisis global, en el que cada grupo de variables es tratado como un ente y permite medir su relación.

- Etapa compromiso: análisis de la tabla global y búsqueda de un espacio de referencia (factores comunes) a todos los grupos analizados

- Etapa de intra-estructura: análisis comparativo de las nubes parciales en el espacio obtenido en la etapa anterior.

\subsection{Método de Clasificación (CLA)}

Por su parte, la metodología CLA se puede resumir en los siguientes puntos:

1. Es un conjunto de métodos que obtiene grupos de observaciones semejantes de acuerdo con algún criterio. Estos objetos están descritos por cierto número de variables o indicadores y es necesario definir una medida de homogeneidad a partir de la cual se detectan los grupos. La clasificación considera dos observaciones semejantes y las asigna al mismo grupo si se parecen en el conjunto de las variables. En el caso que nos ocupa, al tratar con variables cuantitativas, la medida de semejanza será la distancia euclídea.

2. Los métodos de clasificación difieren en la forma de obtención de los grupos: jerárquica ascendente, jerárquica descendente, no jerárquica y mixta. Según el objetivo último y el tipo de variables se elegirá un procedimiento u otro. Los métodos jerárquicos construyen un árbol de clasificación, que puede ser descendente o ascendente, siendo este último el más habitual y el que se va a aplicar en este trabajo. Utiliza el método de agregación de Ward, también llamado de varianza mínima. El proceso consiste inicialmente, en formar tanto grupos como observaciones, posteriormente, y de forma iterativa, el método une los grupos más próximos en el sentido de que su fusión suponga el menor incremento de varianza, esto es, quede garantizada la máxima homogeneidad. De esta forma, se obtiene un árbol de clasificación, denominado dendograma, que describe el proceso de cómo se van uniendo los grupos en cada fase. El árbol, finalmente, se cortará donde surjan las ramas más largas (indicador de mayor heterogeneidad entre los grupos que de ellas "cuelgan") y se definirán tantas clases como las que queden determinadas por las ramas cortadas.

3. Una vez elegida la partición, se obtienen, por un lado, los indicadores de la homogeneidad de las clases obtenidas: la inercia dentro de las clases (inercia intraclase), indicador de la homogeneidad de los grupos y la inercia inter-clase que mide la diferencia entre los mismos. Por otro lado, un listado de las observaciones que pertenecen a cada clase, con gran utilidad en las agrupaciones de observaciones temporales (análisis de la estacionalidad) como en las agrupaciones de contextos geográficos (como se comprobará en la aplicación empírica).

\subsection{Estrategia metodológica para identificar perfiles dinámicos turísticos en diferentes contextos}

La estrategia propuesta se fundamente en la complementariedad de las dos técnicas anteriores, lo que las convierte en una idónea alternativa de exploración de datos (Aluja y Morineau, 1999). Los planos factoriales obtenidos mediante el AFM permiten obtener de forma rápida una "fotografía" de la realidad multivariante objeto de estudio. Suponen, por 
tanto, una excelente aproximación visual a la principal información contenida en los datos tridimensionales. No obstante, esa aproximación es, en general, parcial, debido a que sólo se inspeccionan dos dimensiones a la vez, cuando en realidad el espacio de representación a analizar suele ser de dimensión más elevada. La clasificación, por su parte, es una operación complementaria de síntesis, ya que el posicionamiento de las observaciones sobre el primer plano factorial según su clase de pertenencia constituye un excelente método para poder ver en profundidad más allá de los dos ejes representados y clarificar las representaciones factoriales obtenidas. Este procedimiento tiene, además, otra ventaja: la clasificación sobre los resultados factoriales puede proporcionar resultados más estables, ya que al existir la posibilidad de no conservar todos los ejes (sino sólo los primeros), se eliminará información que recogería fluctuaciones aleatorias que podrían eclipsar fenómenos importantes.

Todo lo comentado anteriormente, la naturaleza espacio-temporal de los datos, su estructuración en tabla múltiple y la complementariedad de las técnicas expuestas, constituyen la hoja de ruta que conduce a la propuesta metodológica. El tándem AFM+CLA no va a proporcionar una simple imagen de la evolución del fenómeno analizado en el tiempo, sino una visión detallada y, sobre todo, cuantificada de los acontecimientos más importantes ocurridos durante el periodo (componente estacional, tendencia, ...) para los diferentes contextos considerados, facilitando su estudio comparativo $y$, por ende, su posible agrupación. A continuación, se presenta esquemáticamente las diferentes etapas que conlleva, cuya forma de proceder, resultados e interpretaciones serán comentados con mayor detalle en el análisis de las diferentes dinámicas provinciales de la demanda turística española, presentado en la siguiente sección. 
Figura 1. Esquema de la estrategia metodológica

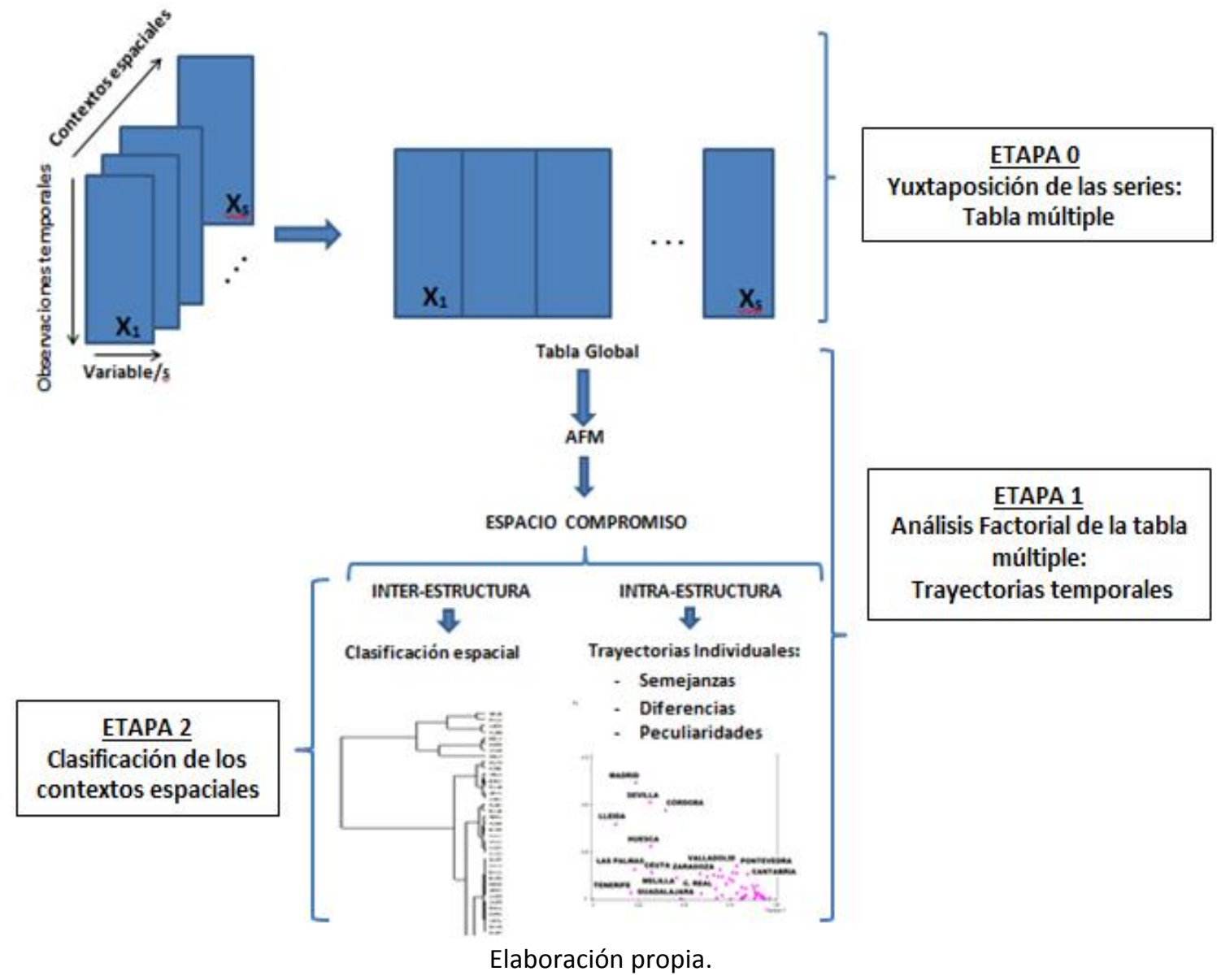

\section{PERFILES DINÁMICOS PROVINCIALES DE LA DEMANDA TURISTICA ESPAÑOLA. PRINCIPALES RESULTADOS}

La etapa inicial del análisis, siguiendo el esquema de la figura 1, y a partir de los datos descritos en la sección 3, conlleva la estructuración de los mismos en una tabla múltiple. Esta tabla global surge de yuxtaponer las 52 series temporales (subtablas unidimensionales), asociadas a cada una de las provincias españolas, respectivamente.

El programa estadístico utilizado ha sido Systéme Pour L'Analyse des Données (SPAD, versión 5.5). No obstante, señalar la disponibilidad de software libre, concretamente el paquete FactoMineR (Le et al., 2008), creado en el seno del Laboratoire de Mathématiques Appliquées, Agrocampus Ouest, Rennes, Francia (se puede consultar más información en http://CRAN.R-project.org/package=FactoMineR).

\section{Etapa 1: AFM de la tabla global - Trayectorias turísticas provinciales}

El AFM ofrece una batería de gráficos y de indicadores numéricos que permiten cuantificar las similitudes y las divergencias entre las series comparadas, matizando qué provincias son las que presentan comportamientos con mayores similitudes, las que se separan en mayor medida de la trayectoria media y en qué momentos de tiempo se producen estos comportamientos. Además, realiza un análisis ponderado, en el que todos los contextos espaciales considerados tienen el mismo peso, independientemente de su "tamaño". Hay que 
adelantar que, al ser grupos de una sola variable, el AFM proporciona ciertos resultados equivalentes a los obtenidos aplicando un ACP, pero notablemente ampliados con el punto de vista adicional de tabla múltiple, con gran utilidad para los objetivos aquí planteados.

Todo esto es posible ya que el AFM proporciona un doble análisis de la tabla de datos: uno global, en el que se analiza la tabla como única (inter-estructura), buscando los factores comunes a todas las subtablas analizadas (compromiso) y otro parcial (intra-estructura) en el que cada mes del periodo se proyecta, en ese espacio compromiso, desde cada uno de los 52 puntos de vista (contextos geográficos) analizados.

Los primeros resultados que merecen ser destacados son aquellos que miden y reflejan un comportamiento global:

- El histograma de los valores propios del análisis de la tabla global (tabla 1): Se comprueba la existencia de un primer eje factorial preponderante que recoge un $71 \%$ de la información inicial, por lo que concluimos la existencia de un patrón principal en la dinámica del turismo presente en muchas de las provincias españolas.

Tabla 1. Primeros valores propios del análisis global ${ }^{1}$

\begin{tabular}{|l|c|c|}
\hline & Valor propio & Porcentaje de inercia \\
\hline $\mathbf{1}$ & 36,95 & 71,05 \\
\hline $\mathbf{2}$ & 5,09 & 9,79 \\
\hline $\mathbf{3}$ & 3.40 & 6,54 \\
\hline $\mathbf{4}$ & 1,76 & 3,39 \\
\hline
\end{tabular}

Elaboración propia a partir de los resultados del programa SPAD.

En lo que respecta al segundo factor, con una inercia de casi un $10 \%$, se puede concluir la existencia de un segundo patrón de dinamismo en la demanda turística, presente en algunas de las provincias del estudio. Los esfuerzos interpretativos, en esta etapa, se van a centrar en el plano principal, ya que con el $80 \%$ de la inercia pone de manifiesto las principales estructuras temporales y espaciales subyacentes en los datos analizados. El resto de los factores reflejan peculiaridades de ciertas dinámicas provinciales; algunas de las cuales, y dados los objetivos, serán asimismo comentadas.

- Plano principal de los grupos, provincias, (figura 2): gráfico en el que cada provincia aparece proyectada a través de un punto en un solo cuadrante. Se trata de una herramienta visual sencilla y muy clarificadora, ya que resume las regularidades y las particularidades de las 52 trayectorias analizadas. La proximidad/distancia entre los puntos-provincia es reflejo de estas semejanzas y diferencias.

\footnotetext{
1 Una combinación de criterios empíricos y estadísticos para la selección del número de ejes a retener, concretamente los desarrollados por Catell, Kayser y los intervalos de confianza de Anderson (Lebart et al., 2006, p. 96-100), conducen a seleccionar los dos primeros ejes, ya que son dominantes y bien caracterizados; para ambos se cumple que el mínimo del intervalo de confianza de un valor propio es superior al máximo del intervalo del siguiente valor propio. Para el tercer y el cuarto factores también se cumple esta regla, por lo que, aunque reflejan poca inercia, también serán analizados algunos de sus indicadores y tenidos en cuenta para la etapa de clasificación.
} 
Este análisis permite vislumbrar las provincias que se alejan del principal perfil dinámico de la demanda turística presente en muchas de las provincias españolas. Los puntos asociados a estás ultimas aparecen proyectados en el extremo del eje horizontal y con pequeña coordenada en el eje vertical. La agrupación de estas provincias en el gráfico se ha realizado únicamente atendiendo a un criterio ilustrativo.

Madrid, Sevilla y Córdoba, con la mayor coordenada en el eje vertical (y, a su vez, una pequeña coordenada en el eje horizontal), presentan un perfil en sus trayectorias turísticas similar entre sí pero diferente al resto. Asimismo, las provincias de Lleida, Huesca, Las Palmas y Tenerife destacan en este primer plano por su posición próxima al origen de coordenadas, posiciones de las que se concluye que son territorios con dinámicas peculiares que habrá que explorar por separado.

Figura 2. Plano principal de las provincias

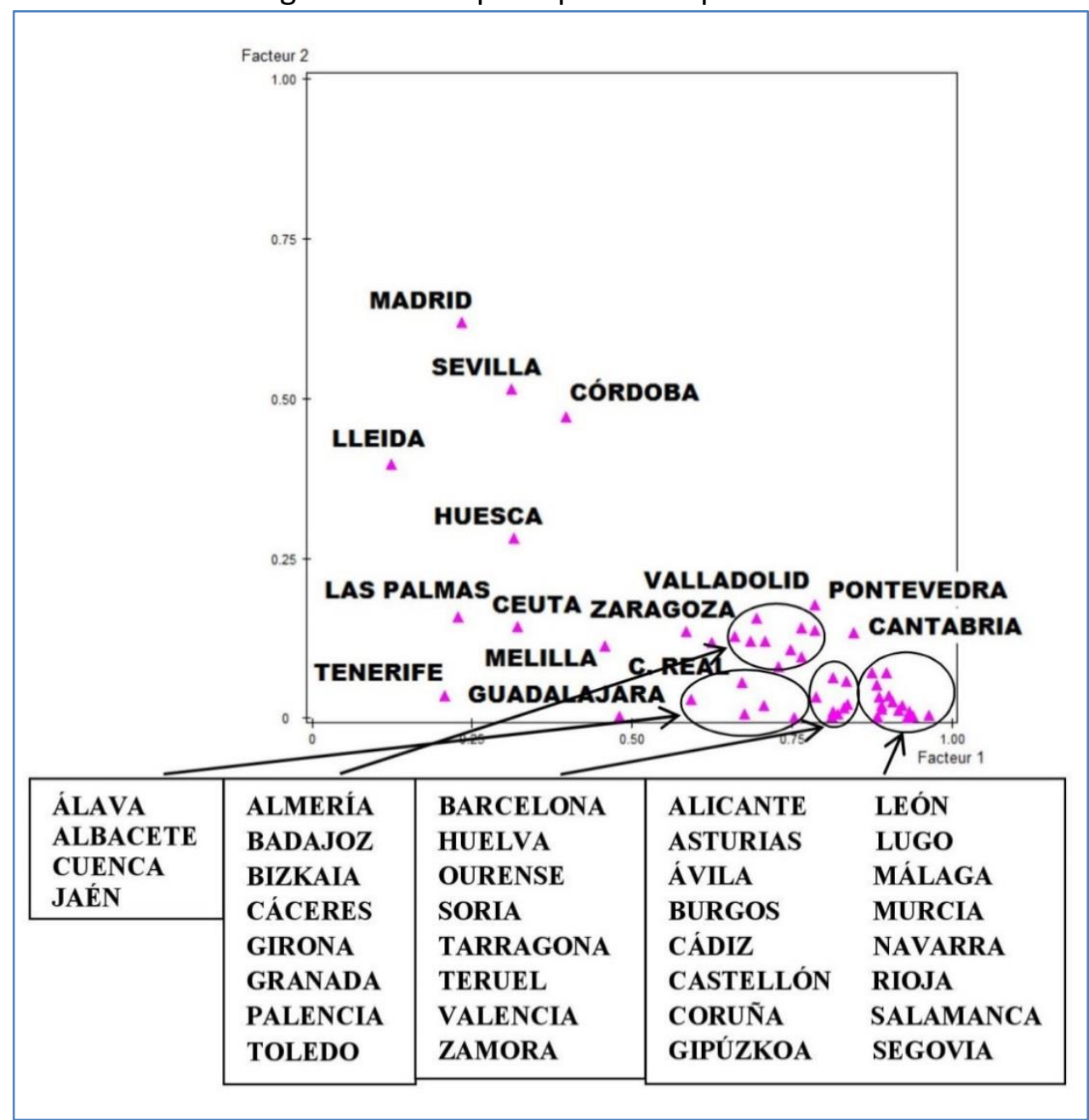

Elaboración propia a partir de los resultados del programa SPAD.

El gráfico en el que queda reflejada la posición de estas provincias en los ejes 3 y 4 , respectivamente, (figura 3), confirma la anterior afirmación. Y, a modo ilustrativo (para no alargar en exceso este trabajo), se presenta también el plano en el que queda reflejada la información del quinto eje, comprobando el protagonismo (casi en exclusiva) de Ceuta (claro reflejo de las destacadas peculiaridades de su perfil turístico). 
La identificación de las distintas trayectorias, que han emergido de todos estos resultados, será posible con la ayuda de otros indicadores, que serán examinados a continuación.

Figura 3. Planos factoriales 1-3, 1-4 y 1-5 de las provincias

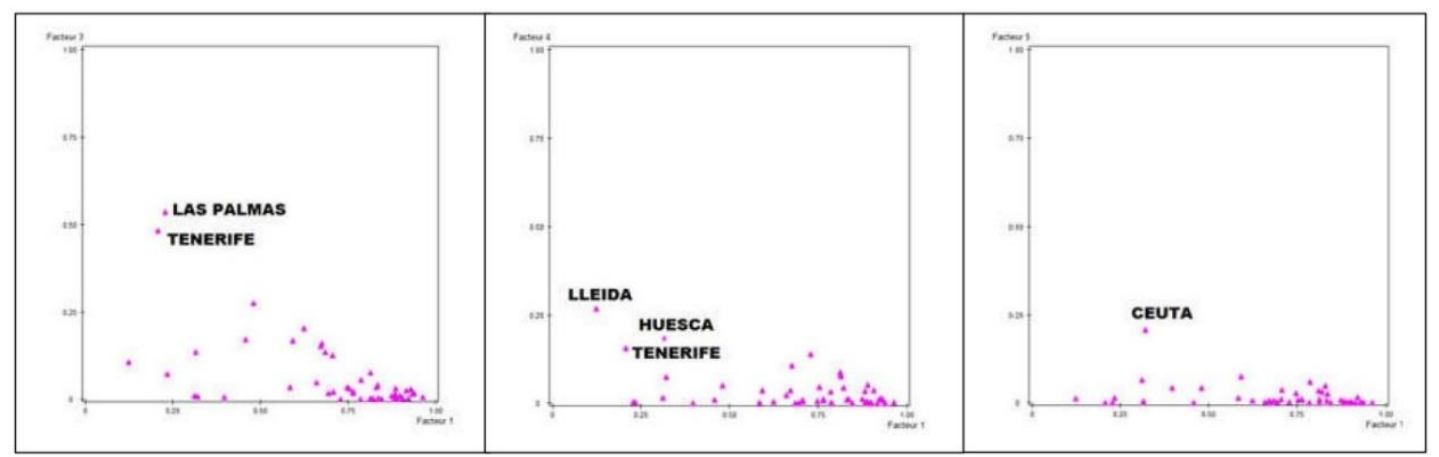

Elaboración propia a partir de los resultados del programa SPAD.

La tabla de las contribuciones de las provincias a la formación de los cuatro primeros ejes (tabla 2) confirma las afirmaciones realizadas hasta el momento; resultando especialmente útil para corroborar el protagonismo que los territorios "peculiares" tienen en los factores 2, 3 y 4, respectivamente.

Estos primeros resultados se centran en la dimensión espacial de los datos y deben ser complementados con el análisis de la dimensión temporal. Este enfoque permitirá identificar el patrón dinámico concreto responsable de las diferencias y similitudes detectadas. A continuación se presentan las herramientas del AFM que permiten avanzar en los objetivos propuestos:

- El plano principal de las observaciones temporales (figura 4), correspondiente al análisis de la "intra-estructura". En este gráfico aparecen proyectados los denominados "puntos medios". En este caso los puntos reflejan la posición media de las observaciones temporales (enero 1999 - septiembre 2016) teniendo en cuenta conjuntamente las 52 subtablas analizadas (provincias). No obstante, hay que tener en cuenta la gran cantidad de información proyectada, 213 observaciones mensuales, lo que conlleva a un plano poco informativo. El voluminoso conjunto de puntos puede ser simplificado a través del uso de variables nominales ilustrativas (Lebart et al., pp 72), lo que permitirá obtener una representación clarificadora de estas observaciones temporales. Como su propio nombre indica, son observaciones o variables que no intervienen de forma activa en el análisis, pero que resultan de gran ayuda en la interpretación de los resultados alcanzados. En este caso, dada la naturaleza de los datos y los objetivos perseguidos, se han definido dos variables ilustrativas ${ }^{2}$ de naturaleza cualitativa. Una de ellas asociada a la dimensión mensual de las observaciones, con 12 modalidades, que ayudará a detectar la posible concentración de la demanda turística en determinados meses, reflejando un posible componente estacional en la misma. Y una segunda variable ilustrativa con 18 modalidades, asociadas a los años analizados respectivamente, que ayudará a vislumbrar

\footnotetext{
2 En las técnicas factoriales es habitual el uso de elementos suplementarios o ilustrativos que consiste en proyectar sobre los factores los perfiles de observaciones o variables que no han intervenido en su obtención, pero que resultan de gran ayuda en su interpretación (Lebart et al., 2005, 72-74).
} 
la posible tendencia en el flujo de las pernoctaciones. La interpretación de los ejes se puede apoyar en la posición de las modalidades ilustrativas siempre que resulten significativas ${ }^{3}$. En este análisis se ha comprobado que los meses han resultado significativos en el primer eje factorial y los años en el segundo.

Tabla 2. Contribuciones de los grupos en los cuatro primeros factores del análisis global

\begin{tabular}{|c|c|c|c|c|c|c|c|c|c|}
\hline \multirow[t]{2}{*}{ PROVINCIA } & \multicolumn{4}{|c|}{ CONTRIBUCIONES } & \multirow[t]{2}{*}{ PROVINCIA } & \multicolumn{4}{|c|}{ CONTRIBUCIONES } \\
\hline & F1 & $F 2$ & F3 & F4 & & $F 1$ & F2 & F3 & $F 4$ \\
\hline G1 - ALMERÍA & 2,1 & 2,8 & 0,5 & 0,7 & G27 - TOLEDO & 2,1 & 1,9 & 0,7 & 0,5 \\
\hline G2 - CÁDIZ & 2,5 & 0,4 & 0,1 & 0,7 & G28 - BARCELONA & 2,2 & 0,2 & 2,2 & 4,4 \\
\hline G3 - CÓRDOBA & 1,1 & 9,3 & 0,2 & 0,0 & G29 - GIRONA & 2,1 & 2,7 & 0,0 & 1,9 \\
\hline G4 - GRANADA & 1,8 & 2,5 & 1,4 & 1,4 & G30 - LLEIDA & 0,3 & 7,8 & 3,1 & 15,3 \\
\hline G5 - HUELVA & 2,3 & 0,3 & 1,0 & 0,6 & G31 -TARRAGONA & 2,2 & 1,3 & 0,1 & 4,6 \\
\hline G6 - JAÉN & 1,8 & 1,1 & 4,5 & 2,2 & G32 - ALICANTE & 2,5 & 0,5 & 0,1 & 2,1 \\
\hline G7 - MÁLAGA & 2,4 & 0,3 & 0,0 & 2,9 & G33 - CASTELLÓN & 2,4 & 1,4 & 0,3 & 0,7 \\
\hline G8 - SEVILLA & 0,8 & 10,1 & 0,3 & 0,9 & G34 - VALENCIA & 2,1 & 0,7 & 1,7 & 0,2 \\
\hline G9 - HUESCA & 0,9 & 5,5 & 4,0 & 10,4 & G35 - BADAJOZ & 1,9 & 2,4 & 0,6 & 0,3 \\
\hline G10 - TERUEL & 2,2 & 0,1 & 0,0 & 5,1 & G36 - CÁCERES & 2,0 & 2,1 & 1,1 & 0,4 \\
\hline G11 - ZARAGOZA & 1,6 & 2,7 & 1,0 & 0,2 & G37 - CORUÑA & 2,4 & 1,0 & 0,0 & 0,0 \\
\hline G12 - LAS PALMAS & 0,6 & 3,1 & 15,8 & 0,2 & G38 - LUGO & 2,5 & 0,2 & 0,7 & 0,0 \\
\hline G13 - TENERIFE & 0,6 & 0,7 & 14,2 & 8,8 & G39 - OURENSE & 2,2 & 0,1 & 0,1 & 2,5 \\
\hline G14 - ÁVILA & 2,4 & 0,0 & 0,3 & 2,0 & G40 - PONTEVEDRA & 2,1 & 3,5 & 0,0 & 0,0 \\
\hline G15 - BURGOS & 2,6 & 0,1 & 0,2 & 0,1 & G41 - ÁLAVA & 2,0 & 0,0 & 0,9 & 2,6 \\
\hline G16 - LEÓN & 2,5 & 0,0 & 0,5 & 0,1 & G42 - BIZKAIA & 1,7 & 2,3 & 6,0 & 0,2 \\
\hline G17 - PALENCIA & 1,9 & 2,4 & 4,0 & 0,0 & G43 - GIPUZKOA & 2,5 & 0,0 & 0,9 & 0,9 \\
\hline G18 - SALAMANCA & 2,4 & 0,7 & 0,9 & 0,3 & G44 - ASTURIAS & 2,4 & 1,4 & 0,3 & 0,1 \\
\hline G19 - SEGOVIA & 2,4 & 0,0 & 0,5 & 0,0 & G45 - BALEARES & 2,0 & 1,6 & 0,0 & 7,9 \\
\hline G20 - SORIA & 2,3 & 0,4 & 0,1 & 0,6 & G46 - CANTABRIA & 2,3 & 2,6 & 0,0 & 0,0 \\
\hline G21 - VALLADOLID & 1,9 & 3,1 & 0,5 & 0,0 & G47 - MADRID & 0,6 & 12,2 & 2,2 & 0,2 \\
\hline G22 - ZAMORA & 2,3 & 1,1 & 1,2 & 0,6 & G48 - MURCIA & 2,4 & 0,4 & 0,0 & 0,2 \\
\hline G23 - ALBACETE & 1,9 & 0,4 & 3,7 & 0,6 & G49 - NAVARRA & 2,5 & 0,2 & 0,5 & 0,5 \\
\hline G24 - C. REAL & 1,6 & 0,6 & 4,9 & 2,1 & G50 - RIOJA & 2,4 & 0,7 & 0,3 & 0,0 \\
\hline G25 - CUENCA & 1,8 & 0,1 & 4,7 & 6,1 & G51 - CEUTA & 0,9 & 2,8 & 0,3 & 4,3 \\
\hline G26 - GUADALAJARA & 1,3 & 0,1 & 8,1 & 2,8 & G52-MELILLA & 1,2 & 2,2 & 5,1 & 0,6 \\
\hline
\end{tabular}

Elaboración propia a partir de los resultados del programa SPAD.

Para una mejor interpretación este plano ilustrativo se ha reflejado en dos gráficos. Así, en la figura 4a se han proyectado las modalidades correspondientes a la variable "mes". A lo largo del eje horizontal (primer factor) se puede observar la existencia de un claro perfil estacional en la evolución del turismo es España, como principal seña de identidad de la trayectoria correspondiente a la mayoría de las zonas estudiadas. También pone de manifiesto los meses de menor y mayor concentración del número de pernoctaciones. En el primer caso destacan los dos últimos y los dos primeros meses de cada año: noviembre, diciembre, enero y febrero. En el segundo caso destacan, por este orden, agosto, julio y septiembre. En la zona

\footnotetext{
${ }^{3}$ La significatividad se mide a través de un v-test que expresa en número de desviaciones tipo de una distribución normal, la diferencia estadística entre la media del grupo de observaciones que poseen la modalidad correspondiente y la media general. Un valor alto indica que el grupo de observaciones que posee dicha modalidad ocupa una posición significativa y caracteriza la zona del plano factorial donde se encuentra En la práctica se utiliza el umbral de 2 desviaciones tipo para considerar la modalidad significativa (Aluja y Morineau, 1999, 68-71).
} 
central del plano quedan situados el resto de los meses, caracterizados por alcanzar valores medios en el indicador estudiado.

Figura 4a. Plano principal de las observaciones temporales.

Proyección de la variable ilustrativa "mes"

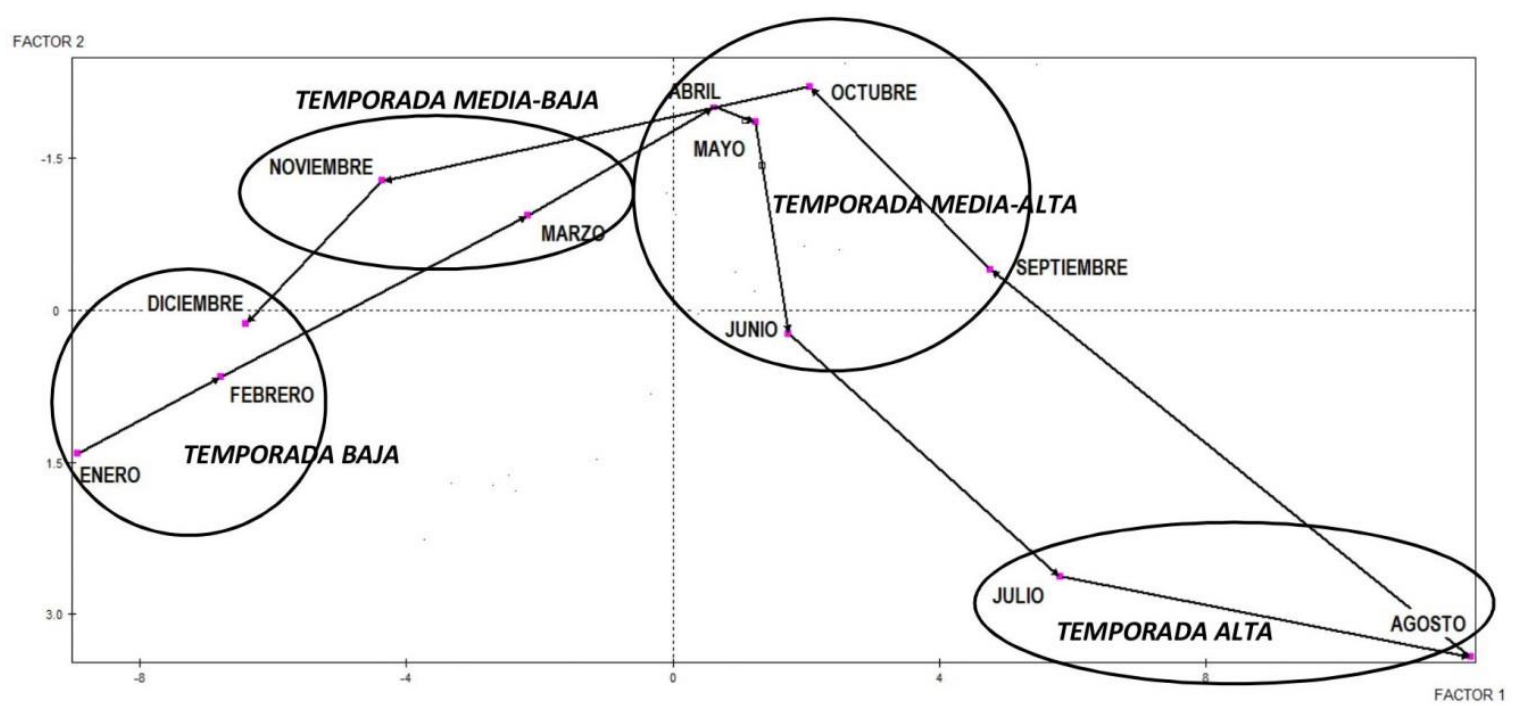

Elaboración propia a partir de los resultados del programa SPAD.

Figura 4b. Proyección de la variable ilustrativa "año"

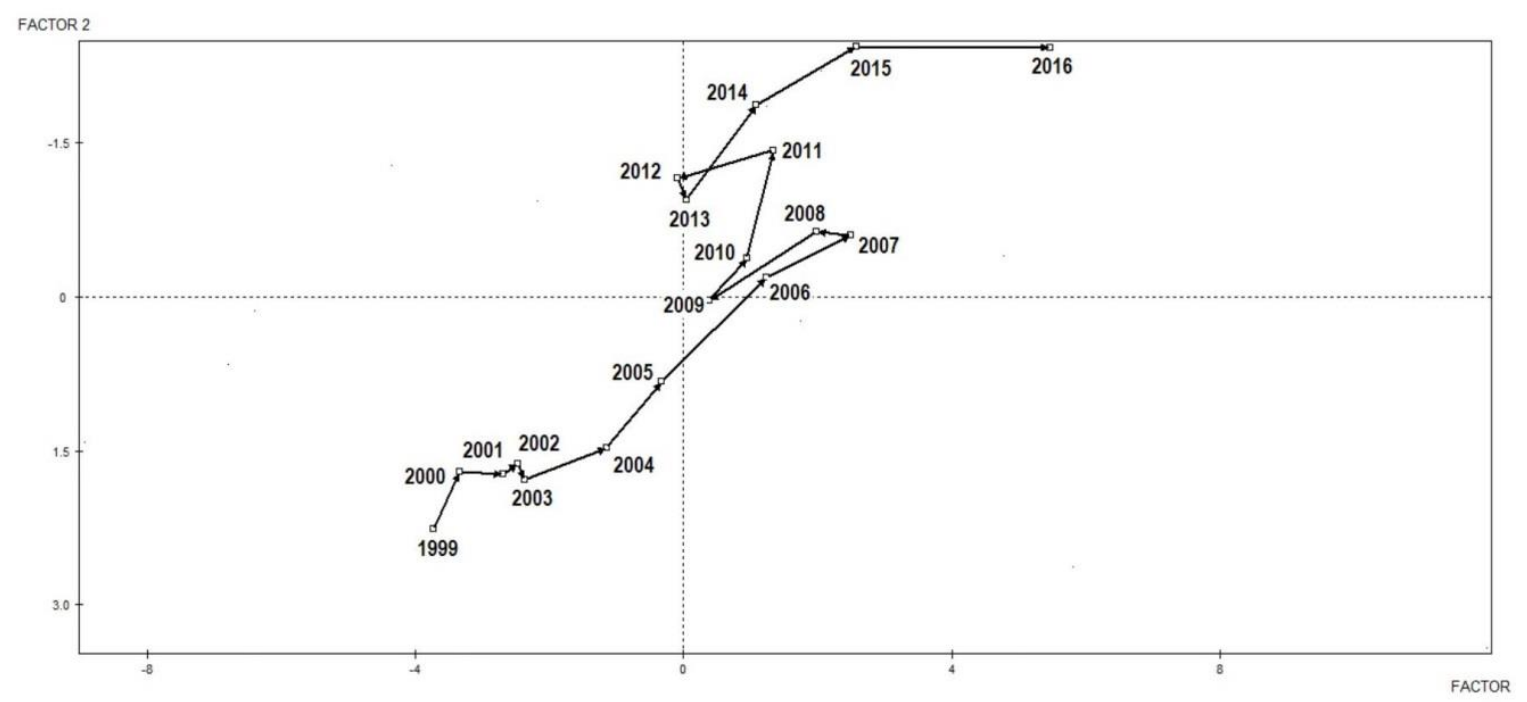

Elaboración propia a partir de los resultados del programa SPAD.

En la figura $4 \mathrm{~b}$ se han proyectado las modalidades correspondientes a la variable "año", con protagonismo en el eje vertical, reflejando la existencia de un segundo patrón de dinámica tendencial alcista, presente en el perfil turístico de algunas de las provincias estudiadas. La posición de los puntos-año refleja, en cierta medida, como en el sector turístico la senda del crecimiento económico se ha ido consolidando. Apreciándose, asimismo, como la actividad turística no ha permanecido ajena a la 
importante crisis económica sufrida, como queda reflejado en el retroceso de la línea de tendencia en 2008 y especialmente en 2009.

Estas primeras conclusiones alcanzadas serán matizadas, confirmadas y ampliadas con otros resultados propios del AFM, correspondientes a la dimensión espacial de la información, y a los obtenidos en la etapa de clasificación.

Una herramienta numérica adicional del AFM, que matiza las evoluciones peculiares antes comentadas, es la relación de los puntos parciales con las mayores inercias intra (tabla 3). Esto es, las observaciones mensuales de años concretos que se alejan, en mayor medida, del comportamiento medio de cada trayectoria individual y, por tanto, responsables de las diferencias que presentan algunas provincias. Un rápido recorrido por dicha relación, para los factores segundo, tercero y cuarto, permite comprobar la asociación entre las observaciones peculiares con los territorios ya señalados como protagonistas en la formación de los mismos.

Tabla 3. Individuos parciales con mayor inercia intra: observaciones temporales peculiares

\begin{tabular}{|l|l|l|l|}
\hline \multicolumn{1}{|c|}{ Factor 1 } & \multicolumn{1}{|c|}{ Factor 2 } & \multicolumn{1}{c|}{ Factor 3 } & \multicolumn{1}{c|}{ Factor 4 } \\
\hline Enero - 1999 - Ceuta & Octubre - 2015 - & Agosto - 2016 - Las Palmas & Agosto - 2016 - Huesca \\
Agosto - 2008 - Zaragoza & Madrid & Agosto - 2016 - Tenerife & Agosto - 1999 - Lleida \\
Julio - 2008 - Zaragoza & Agosto - 2008 - & Julio - 2016 - Las Palmas & Agosto - 2001 - Lleida \\
Febrero - 1999 - Ceuta & Zaragoza & Agosto - 2015 - Las Palmas & Agosto - 2005 - Lleida \\
Enero - 2014 - Tenerife & Mayo - 2016 - Sevilla & Agosto - 2014 - Las Palmas & Agosto - 2000 - Lleida \\
Abril - 2011 - Badajoz & Abril - 2016 - Sevilla & Julio - 2016 - Tenerife & Agosto - 2006 - Lleida \\
Agosto - 2015 - Guadalajara & Agosto - 2016 - Huesca & Agosto - 2015 - Tenerife & Agosto - 2004 - Lleida \\
Agosto - 2014 - Guadalajara & Mayo - 2015 - Madrid & Julio - 2015 - Las Palmas & Agosto - 2016 - Lleida \\
Agosto - 2011 - Zamora & Mayo - 2015 - Córdoba & Agosto - 2007 - Tenerife & Agosto - 2015 - Huesca \\
Agosto - 2006 - Madrid & Mayo - 2016 - Córdoba & Agosto - 2016 - Bizkaia & Agosto - 2007 - Lleida \\
& Agosto - 2016 - Ceuta & & \\
& Octubre - 2015 - Sevilla & & \\
& &
\end{tabular}

Elaboración propia a partir de los resultados del programa SPAD.

Una atención especial merece los puntos parciales en el primer factor. Para comprobar la utilidad de esta herramienta en la detección de observaciones atípicas en las trayectorias, se ha completado su presentación con la figura 5. En ella se reflejan los perfiles completos de las cuatro primeras provincias con mayor inercia intra, para ilustrar y facilitar la identificación de estas observaciones ( $y$ en su caso, tratar de encontrar una justificación). Se puede comprobar cómo Ceuta comienza la serie (enero y febrero de 1999) con unos valores de pernoctaciones anormalmente altos. Zaragoza, asimismo, presenta unos valores en julio y agosto de 2008 extraordinariamente superiores a su evolución media (siendo fácilmente identificables, ya que esta provincia fue la encargada de organizar la Exposición Universal de 2008). Tenerife, por su parte, presenta un pico en enero de 2014 que se aleja del comportamiento medio en esa época del año. Al igual que le ocurre a Badajoz en abril de 2011. 
Figura 5. Dinámicas turísticas con observaciones peculiares

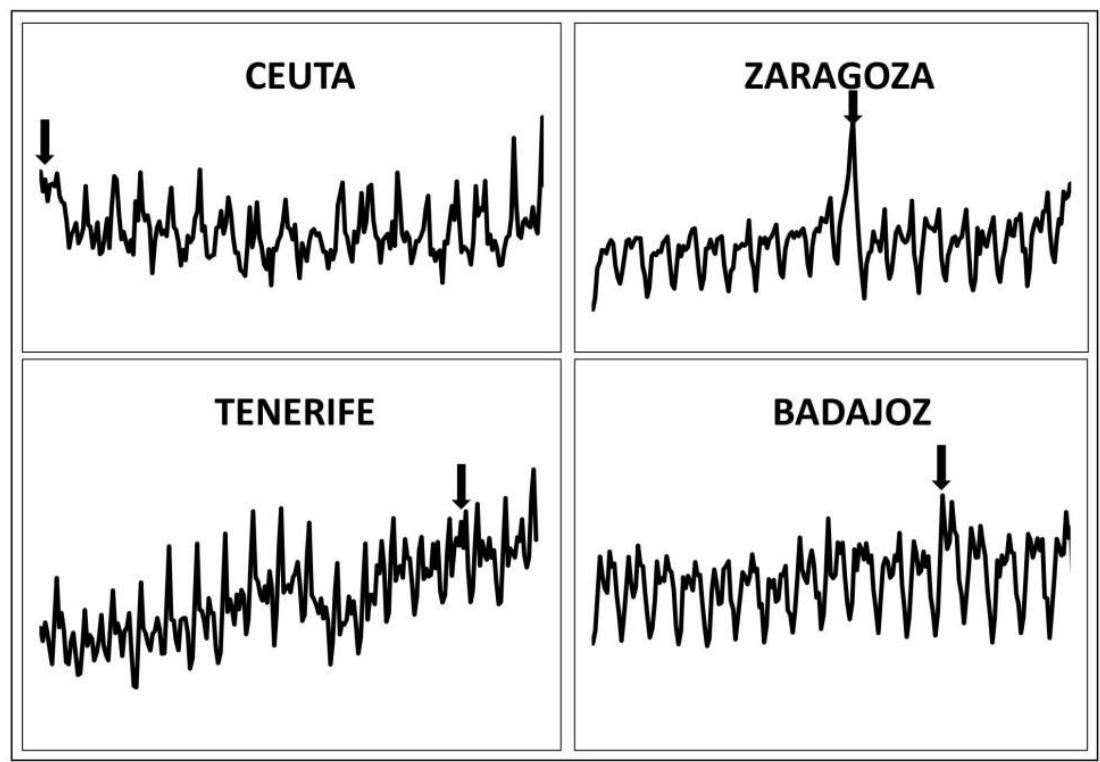

Elaboración propia a partir de los resultados del programa SPAD.

La etapa 1 de esta propuesta metodológica ha permitido aflorar e identificar regularidades en las dinámicas turísticas del conjunto de provinciales españolas, con diferentes patrones de concentración temporal. Asimismo, los resultados alcanzados en esta etapa han proporcionado indicios de las posibles agrupaciones de provincias en función de las similitudes en sus trayectorias. No obstante, la etapa 2 de clasificación resulta conveniente y necesaria para definir con mayor rigor las diversas tipologías existentes en la geografía española.

\section{Etapa 2: CLASIFICACIÓN - Tipologías provinciales según dinámica turística}

A partir de la información analizada, de las conclusiones obtenidas y de los resultados seleccionados en la etapa anterior, se alcanza la etapa final de la estrategia metodológica propuesta: clasificación de las provincias españolas atendiendo al perfil de su dinámica turística. La técnica usada en esta fase ya ha sido comentada en la sección 2: Método de Clasificación Jerárquica Ascendente. Únicamente recordar que se trata de un método de clasificación no supervisado, basado en la distancia euclídea y en el criterio de Ward.

La agrupación de las provincias se va a realizar a partir de sus coordenadas en los cuatro primeros factores del AFM de la tabla global, que, como se ha comprobado en la etapa anterior, reflejan los principales rasgos de sus trayectorias. Esta decisión supone una gran ventaja al disminuir la parte de "ruido" que los datos originales contienen. 
Figura 6. Clasificación de las provincias según sus trayectorias. Dendograma

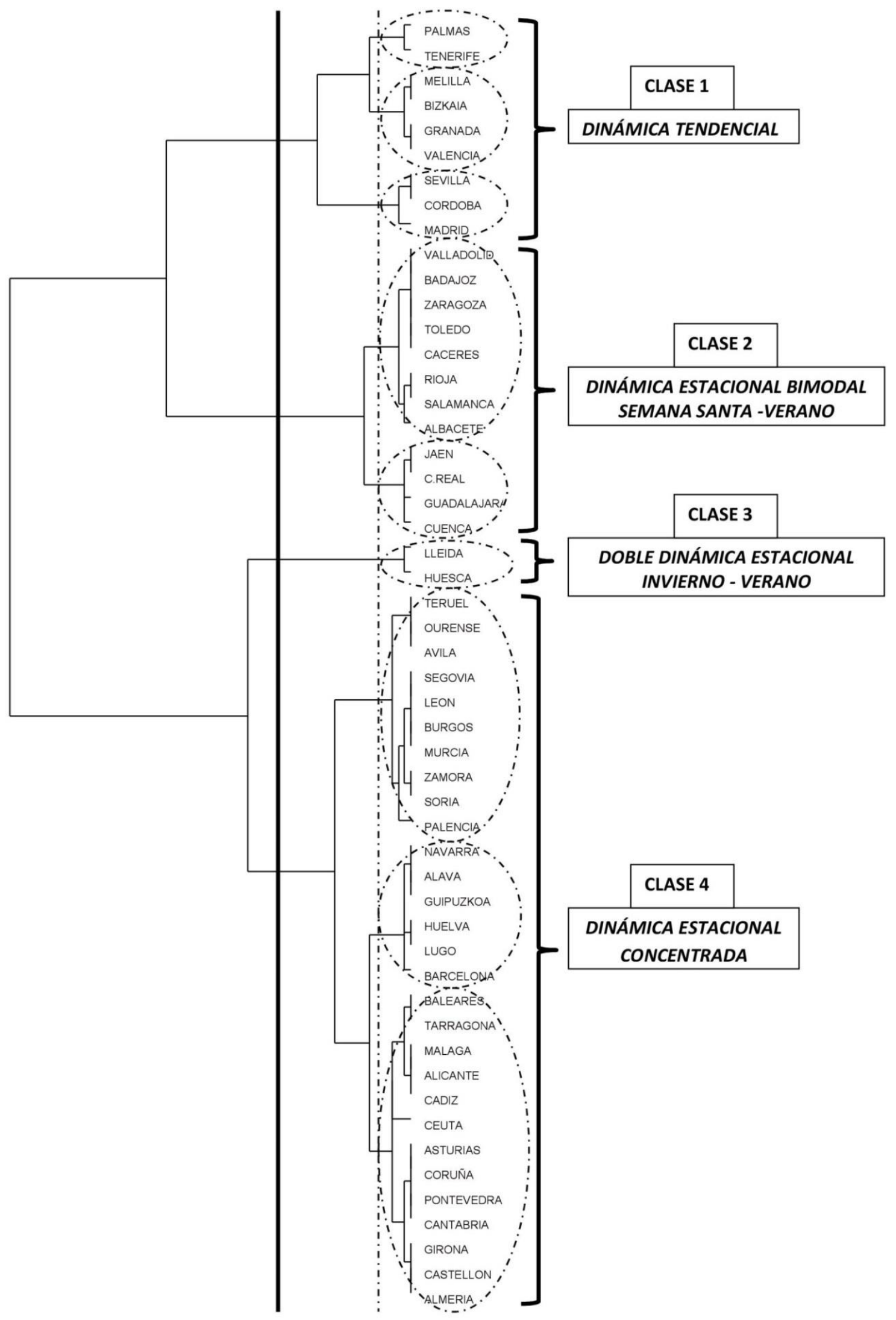

Elaboración propia a partir de los resultados del programa SPAD. 
La principal herramienta visual para la definición de las diferentes tipologías es el dendograma (figura 6). En la figura 7 se recogen los perfiles dinámicos de los parangones ${ }^{4}$ de cada uno de los cluster, lo que supone una ayuda para la interpretación de la trayectoria definida por cada uno de ellos. A partir de estos gráficos, y con la ayuda de indicadores numéricos apropiados ${ }^{5}$, se concluye que el corte del árbol en cuatro "ramas" resulta adecuado en este caso (corte señalado en el gráfico a través de la línea continua); identificando, como puede comprobarse, cuatro patrones diferentes:

- Clase 1. Integrada por siete unidades espaciales: Las dos provincias canarias, Melilla, Bizkaia, Granada, Valencia, Sevilla, Córdoba y Madrid. El parangón o representante de este grupo es Melilla (figura 7), cuyo perfil unido al hecho de que las tres últimas provincias antes mencionadas son las protagonistas del segundo factor (eje de tendencia), llevan a la conclusión de que se trata de un cluster cuya actividad turística está caracterizada principalmente por presentar una dinámica tendencial. Hay que señalar que nos encontramos ante un grupo con cierta heterogeneidad (con ramas largas por debajo del corte).

- Clase 2. Integrada por doce territorios: las dos provincias de Extremadura, Castilla- La Mancha al completo y La Rioja, además de las provincias de Jaén, Valladolid, Salamanca y Zaragoza. Su parangón es la provincia de Salamanca, cuyo perfil (figura 7) refleja un característico patrón de concentración de la demanda turística con dos picos, el mayor en la época estival y un segundo (algo menor) en Semana Santa. Se trata de un cluster cuya actividad turística está caracterizada principalmente por presentar una dinámica estacional bimodal.

- Clase 3. Es la más homogénea, debido a que está integrada únicamente por las dos provincias pirenaicas, con un patrón muy similar entre sí, pero con peculiaridades frente al resto (recordar que son claras protagonistas en el cuarto factor). El perfil de Huesca (figura 7), pone claramente de manifiesto la existencia de dos temporadas turísticas diferenciadas coincidentes con las épocas de esquí y estival, respectivamente. El diferente nivel de concentración de la demanda turística entre ambas épocas es notable, por lo que no se puede hablar, como en la clase anterior, de dinámica estacional bimodal, por lo que la hemos denominado doble dinámica estacional.

- Clase 4. Es la más numerosa (veintinueve unidades espaciales) y por ende la más heterogénea. La mayor parte de las provincias costeras se encuentran agrupadas en esta clase, aunque también lo conforman numerosos territorios de interior. La región de Castilla y León, exceptuando Salamanca y Valladolid, está muy bien representada en esta agrupación. El perfil del parangón del clúster (figura 7), Cádiz, refleja un patrón típico con extraordinaria concentración en los meses estivales. Recordar que este rasgo ha quedado reflejado, como no podía ser de otra manera por su destacada

\footnotetext{
${ }^{4}$ Parangones son los elementos más representativos (más próximos al centro) de cada clúster.

5 Índices de nivel que miden la disimilaridad entre las clases, proceso de consolidación alrededor del centro de cada grupo (método de centros móviles) y descomposición de la inercia antes y después de la consolidación para cada clase (Lebart y otros, 2006, 311-318).
} 
presencia en el territorio nacional, en el primer eje factorial. Por lo que se confirma que la trayectoria turística provincial más común en España se caracteriza por tener una dinámica estacional concentrada.

Figura 7. Provincias parangones: trayectorias representantivas de las clases

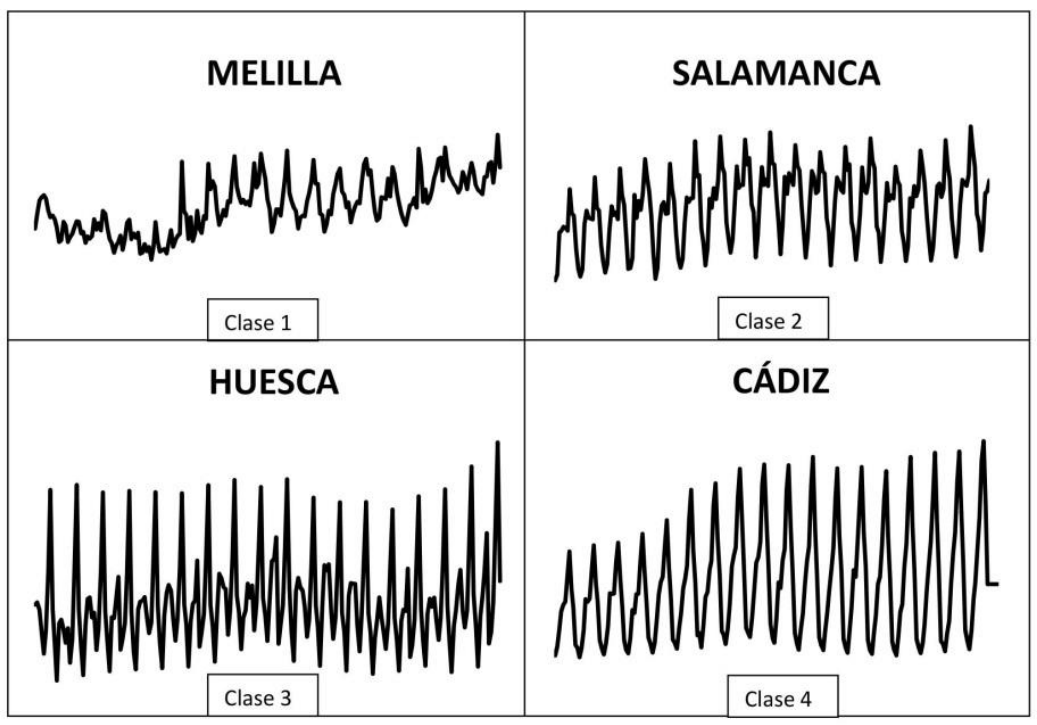

Elaboración propia a partir de los resultados del programa SPAD.

La riqueza del análisis exploratorio de datos radica en que el investigador juega un papel fundamental en la interpretación de los resultados aportados por las diferentes técnicas aplicadas, con base en la ambición de sus objetivos. En esta investigación esta riqueza queda patente en la posibilidad de proceder a una clasificación más "afinada" de las provincias españolas según sus trayectorias de actividad turística. En este sentido, en el dendograma (figura 6) se ha señalado, con líneas discontinuas, una alternativa de partición en nueve clases (más homogéneas en su composición), lo que conllevaría una identificación más exhaustiva de las peculiaridades de los perfiles provinciales. No obstante, se reitera la adecuación de las cuatro clases definidas, dados los objetivos planteados en este artículo, resultando esta segunda clasificación una ayuda para visualizar la mayor o menor homogeneidad de los cluster definidos.

\section{DISCUSIÓN}

El enfoque exploratorio espacio-temporal para la identificación de los perfiles dinámicos provinciales de la demanda turística nacional, desarrollado en este trabajo, ha permitido dar respuesta a todas las preguntas planteadas:

¿La dinámica turística, desde el punto de vista de la demanda, presenta un perfil estacional? ¿Esta dinámica ha sido similar en los 52 territorios considerados? La metodología propuesta ha confirmado que el componente estacional es la principal seña de identidad del perfil turístico a nivel provincial, pero no de forma única. Así, por un lado, para ciertos territorios se ha identificado la tendencia como primer rasgo característico. Y, por otro lado, los periodos de mayor y menor concentración turística no son coincidentes en todas las provincias (identificando turismo de sol y playa, turismo de invierno y turismo de Semana Santa). 
¿Qué provincias mantienen las mayores similitudes? ¿Y las mayores diferencias? La etapa 1 de la propuesta de análisis, el análisis factorial exploratorio, ha proporcionado diferentes señales, gráficas y numéricas, sobre qué provincias presentan evoluciones turísticas similares y sobre aquellas que se alejan de estas similitudes. No obstante, es la etapa 2, clasificación, la que proporciona las herramientas de análisis más rigurosas para la identificación y validación de los diferentes patrones dinámicos turísticos. Hay que señalar que son, en última instancia, el experto en la realidad a analizar y los objetivos últimos de la investigación los verdaderos artífices de la clasificación "final", ya que las alternativas pueden ser diversas.

¿En qué unidades temporales se han producido estas similitudes y/o diferencias? Para la identificación de diferentes "temporadas" en el seno de las series estudiadas, comunes entre algunos territorios, el uso de elementos suplementarios (variables año y mes) en la etapa factorial ha tenido un papel protagonista. Asimismo, la técnica propuesta en esta etapa también ofrece indicadores de los momentos puntuales (un determinado mes de un año concreto) en los que algunas provincias presentan comportamientos peculiares. Estos hallazgos resultan de gran utilidad para posibles estudios posteriores con objetivos confirmatorios, explicativos y/o predictivos.

La estrategia de análisis se ha propuesto siguiendo un protocolo ordenado de sucesivas etapas. No obstante, como se ha podido comprobar a través de la investigación empírica, todos los resultados están, de alguna manera, interrelacionados y no deben interpretarse de manera aislada. Todos ellos deben ser tenidos en cuenta para alcanzar y validar las conclusiones finales.

\section{CONCLUSIONES Y FUTURAS LÍNEAS DE INVESTIGACIÓN}

La originalidad y exhaustividad de esta investigación se ha basado en el doble enfoque "espacio-temporal" de la información analizada: las pernoctaciones hoteleras mensuales en las 52 provincias de la geografía nacional, quedando identificados diferentes perfiles provinciales. La estrategia metodológica, centrada en el "aprendizaje de los datos" y combinando la estructuración de la información en tres dimensiones, el análisis factorial (AFM) y los métodos de clasificación (CLA), ha resultado idónea para alcanzar con éxito los objetivos planteados. Esta propuesta presenta las siguientes ventajas:

- El distinto tamaño (en volumen de turistas) de las unidades espaciales no supone ningún inconveniente para los objetivos planteados, ya que el AFM, al igual que las técnicas factoriales clásicas, pondera la influencia de las variables analizadas conjuntamente. En este caso, la ponderación también se extrapola a la nueva dimensión de grupos propia de esta técnica de análisis de tablas múltiples.

- El AFM proporciona planos factoriales en los que aparecen reflejadas desde una óptica distinta las trayectorias turísticas provinciales. Por una parte, desde un punto de vista global, y con base en la estructuración en grupos de las series yuxtapuestas, permite ver en un solo gráfico todas las series representadas a través de un solo punto cada una. Este gráfico ofrece una imagen simplificada de los territorios con mayores similitudes, las dinámicas peculiares, etc. Por otra parte, permite proyectar en un solo gráfico las series completas, obteniendo una imagen global de las unidades temporales 
en las que se producen tanto las semejanzas como las diferencias. El uso de los denominados elementos ilustrativos resulta de gran ayuda cuando se trabaja con series voluminosas, como en este caso.

- En la interpretación de estos gráficos del AFM no existe el riesgo de caer en subjetividades, ya que a su vez proporciona una serie de indicadores numéricos que permiten identificar y cuantificar las similitudes y las divergencias entre las trayectorias. En este sentido, también proporciona resultados que matizan en qué momentos de tiempo se producen comportamientos extraordinariamente peculiares.

- La etapa de clasificación (CLA) también proporciona asimismo resultados gráficos y numéricos, con objetivos propios, pero que, además, corroboran y, por tanto, avalan las conclusiones alcanzadas en la etapa anterior. El examen de estos resultados capacita al investigador para definir una agrupación de las provincias, en un número determinado de clases, según el nivel de concentración del flujo turístico. Lo que conlleva una verificación de los diversos tipos de perfiles dinámicos de la demanda turística existentes a lo largo de la geografía española, anteriormente identificados.

La aplicación de esta estrategia a las pernoctaciones hoteleras mensuales, correspondientes a los últimos 18 años, en el conjunto de provincias de la geografía nacional ha permitido identificar los patrones y las concentraciones temporales subyacentes en las trayectorias turísticas, obteniendo finalmente una clasificación en cuatro diferentes tipologías:

- Provincias que se caracterizan por presentar una clara dinámica estacional en su actividad turística, con gran concentración en los meses estivales. Es el grupo más numeroso y está integrado básicamente por la mayoría de provincias costeras, la región gallega, siete de las provincias castellano-leonesas (exceptuando Valladolid y Salamanca), Navarra, Álava y Guipúzcoa y Teruel.

- Provincias que se caracterizan por tener un perfil estacional bimodal, presentando, además de un pico en los meses de verano, otro en la época de Semana Santa. A este grupo pertenecen las regiones de Extremadura, Castilla- La Mancha y La Rioja, además de las provincias de Jaén, Valladolid, Salamanca y Zaragoza.

- Provincias que se caracterizan principalmente por su dinámica tendencial: Comunidad Canaria, Melilla, Bizkaia, Granada, Valencia, Sevilla, Córdoba y Madrid. Es un grupo con comportamientos peculiaridades en su composición.

- Provincias que se caracterizan por lo que hemos denominado doble dinámica estacional, ya que presentan una gran concentración turística en la época estival y a su vez una menor, pero destacada, concentración en los meses de invierno: Lleida y Huesca.

No obstante, y a pesar de haber alcanzado el objetivo nuclear, este trabajo adolece de una serie de limitaciones que, a su vez, se erigen como futuras líneas de investigación. La dinámica turística es un fenómeno multidimensional. En esta investigación, siguiendo la estela de trabajos anteriores, se ha analizado únicamente desde el punto de vista de la demanda y a 
través de un solo indicador (pernoctaciones). En este sentido, sería conveniente ampliar el estudio en diferentes direcciones:

- Una primera línea supondría la incorporación de otros factores que se relacionan con el perfil estacional, pudiendo ser estos factores de naturaleza no métrica. La metodología propuesta en este trabajo se puede aplicar a datos categóricos. Como se ha adelantado en la presentación, el AFM permite el estudio de variables nominales (e incluso de datos mixtos, siempre que las variables que integran cada grupo sean de la misma naturaleza). Esta característica abre la posibilidad de completar el análisis de los factores relacionados con la concentración de los flujos turísticos incorporando factores cualitativos (localización geográfica, zona climática...) y trabajar con tablas múltiples mixtas (Abascal y otros, 2006).

- Una segunda línea podría centrarse en el desglose de la demanda turística atendiendo a diferentes criterios (nacionalidad, tipo de turismo, etc.), continuando con el enfoque de Muñiz en su trabajo de 2001.

- Y una tercera línea estaría relacionada con la incorporación de indicadores relacionados con la oferta turística, continuando la investigación de López y López (2006).

\section{REFERENCIAS BIBLIOGRÁFICAS}

Abascal E., García-Lautre I. y Landaluce M.I. (2004). Análisis de la evolución a través de encuestas. Trayectoria electoral de las comunidades autónomas españolas en el periodo 1977-2004. Metodología de Encuestas, 6 (2), 147-162

Abascal E. y Grande I. (2005). Análisis de Encuestas. ESIC. Madrid

AbascaL, E., García-Lautre I. y Landaluce, M. I. (2006). Multiple factor analysis of mixed tables of metric and categorical data. En J. Blasius \& M. Greenacre (Eds.), Multiple Correspondence Analysis and related Methods (351-367). Chapman \& Hall (TAYLOR \&FRANCIS GROUP).

Aguiló, E. (1984) La medición de la estacionalidad del turismo: el caso de Baleares, Estudios Turísticos, 81, 79-88.

Aguiló, E. y Antón, S. (Coords.) (2015). 20 retos para el turismo en España. Pirámide. Madrid

Albacete, C.A. y Fuentes Sáez, M.M. (2009) Difusión de la investigación española sobre turismo en revistas internacionales, XIV Congreso anual de AECIT, Gijón

Aluja, T y Morineau A. (1999). Aprender de los datos: El Análisis de Componentes Principales. Una aproximación desde el Data Mining. EUB. Barcelona

Baron, R.V. (1975). Seasonality in Tourism - a Guide to de Analysis of Seasonality and Trends for Policy Making. Technical Series. 2. The Economist Intelligence Unit Ltd: London

Bujosa, A. y Roselló, J. (2011). Cambio climático y estacionalidad turística en España: Un análisis del turismo doméstico de costa, Estudios de Economía Aplicada, 29 (3), 863880.

Cisneros-Martínez, J.D. y Fernández-Morales, A. (2015). Cultural tourism as tourist segment for reducing seasonality in a coastal area: the case study of Andalusia. Current Issues in Tourism, 18, 765-784 
Dazy, F. y Le Barzic, J.F. (1996). L'Analyse des Données Evolutives. Technip. Paris

Duro, J.A y Farré, F.X (2015). Estacionalidad turística en las provincias españolas: Medición y Análisis. Cuadernos de Turismo, no 36, 157-174

Escofier, B. y Pagès, J. (1986). Le traitement des variables qualitatives et tableaux mixtes par analyse factorielle multiple. Data Analysis and Informatics, IV(2), 179-191

Escofier, B. y Pagès, J. (1992). Análisis factoriales simples y múltiples. Objetivos, métodos e interpretación. Servicio editorial de la Universidad de País Vasco

Escofier, B. y Pagès, J. (1994). Multiple factor analysis (AFMULT package). Computational Statistics \& Data Analysis 18, 121-140. North-Holland

EXCELTUR. Alianza para la excelencia turística. Informe Perspectivas Turísticas, 58, Octubre (2016).

García-Lautre, I. y Abascal, E. (2003). Una metodología para el estudio de la evolución de variables latentes. Análisis de las infraestructuras de carreteras de las comunidades autónomas (1975-2000). Estadística Española, 45, № 153, 193-210.

Koenig-Lewis, N. y Bischoff, E. (2005). Seasonality research: the state of the art. International Journal of Tourism Research 7(4-5), 201-219.

Landaluce, M. I. (1995). Estudio de la estructura de gasto medio de las Comunidades Autónomas españolas. Una aplicación del Análisis Factorial Multiple (Tesis Doctoral). Universidad del País Vasco

Landaluce, M. I. (2016). Análisis Exploratorio de Estructuras Temporales desde la óptica de Tablas Múltiples. Una aplicación. Métodos Cuantitativos para la Economía y la Empresa, 22, 55-77

Landaluce, M. I. (2017). Enfoque exploratorio multivariante para el análisis de estructuras temporales. Una aplicación a la evolución de la demanda turística en España. Estudios de Economía Aplicada, 32 (2), 465-492

Le, S., Josse, J. y Husson, F. (2008). FactoMineR: An R Package for Multivariate Analysis. Journal of Statistical Software, 25(1), 1-18.

Lebart, L., Piron, M y Morineau, A. (2006). Statistique Exploratoire Multidimensionnelle. Visualisation et inférence en fouilles de données. Dunod. Paris. 4e édition

Lopez, J. M. y Lopez L. M. (2006). La concentración estacional en las regiones españolas desde una perspectiva de la oferta turística. Revista de estudios regionales, 77, 77-104.

Lopez, J. M. y Lopez L. M. (2007). Variabilidad estacional del mercado turístico en Andalucía. Estudios y Perspectivas en Turismo, 16, 150-172.

Muñiz, D. (2001). El turismo social como tipología turística desestacionalizadora de la empresa hotelera. Cuadernos de CC.EE. y EE., 41, 93-109.

Ramón, A.B. y Abellán, M.J. (1995) Estacionalidad de la demanda turística en España. Papers de Turisme 17, 47-74.

Riera, A. Ripoll, A.M. y Juaneda, C.N. (2011). Efficiency and Seasonality in the Balearic Hospitality Industry, Estudios de Economía Aplicada 29 (3), 845-862.

Robert, P. y Escoufier, Y. (1976). A Unifying Tool for Linear Multivariate Statistical Methods: The RV-Coefficient. Applied Statistics 25 (3), 257-265.

SECRETARIA GENERAL DE TURISMO (2007). Plan del Turismo Español. Horizonte 2020 Ministerio de Industria, Comercio y Turismo.

SPAD v5.5 (2000). Systéme Portable d'Analyse des Donées Numeriques, CISIA, Montreuil, France. 
Wanhill, S. (2011). What Tourism Economist Do. Their Contribution to Understanding Tourism, Estudios de Economía Aplicada, 29(3), 679-692.

WTTC (2016). Travel \& Tourism. Economic Impact. World Travel \& Tourism Council 